\title{
Competências dos Profissionais da Informação relacionadas à inovação a partir da produção científica
}

\author{
Priscila Machado Borges Sena ${ }^{1}$, Ana Paula Alves Soares ${ }^{2}$, José Antonio Moreiro González ${ }^{3}$, Ursula \\ Blattmann $^{4}$
}

1 ORCID: 0000-0002-5612-4315 Programa de Pós-Graduação em Ciência da Informação da Universidade Federal de Santa Catarina, Florianópolis, Santa Catarina. priscilasena.ufsc@gmail.com. 2 ORCID: 0000-0001-7318-1170 Programa de Pós-Graduação em Ciência da Informação da Universidade Federal de Santa Catarina, Florianópolis, Santa Catarina. ap_as@hotmail.com.

${ }^{3}$ ORCID: 0000-0002-8827-158X. Universidad Carlos III de Madrid. jamore@bib.uc3m.es.

4 ORCID: 0000-0002-8834-0987. Programa de Pós-Graduação em Ciência da Informação da Universidade Federal de Santa Catarina, Florianópolis, Santa Catarina. ursula.blattmann@ufsc.br.

Tipo de contribuição: Comunicação oral

Palavras-chave: Competências dos profissionais da informação; Inovação; Informação e inovação.

\section{Introdução}

O contexto da sociedade do século XXI pauta-se em quase totalidade nas constantes inovações tecnológicas. Refletir sobre as competências dos profissionais da informação ante essa realidade torna-se primordial, pois estes quando não estão inseridos em unidades de informação convencionais (arquivos, bibliotecas e museus), encontram-se em organizações que compreendem a informação e o conhecimento como subsídio essencial para a obtenção de destaque econômico e social.

Em consonância com a linha temática "5 Formação universitária em informação e documentação: avaliação, inovação e horizontes de futuro", objetiva-se neste trabalho investigar a produção científica acerca das competências dos profissionais da informação relacionadas à inovação. A investigação ocorreu a partir da produção científica em Ciência da Informação nas bases de dados Web of Science (WoS), Scopus, Library, Information Science \& Technology Abstracts (LISTA), Library and Information Science Abstracts (LISA).

Deste modo, na próxima seção são descritos os procedimentos metodológicos utilizados para o alcance do objetivo estabelecido, bem como na sequência são apresentados os resultados e discussão que levam as considerações finais, última seção deste estudo.

\section{Caminhos metodológicos}

Caracteriza-se este trabalho como uma pesquisa exploratória descritiva partindo de um estudo bibliométrico dos dados recuperados em bases de dados internacionais. A sistematização das buscas ocorreu por meio do método SystematicSearchFlow (SSF) de Ferenhof e Fernandes (2016).

O método SSF possui 4 fases (Protocolo de Pesquisa, Análise, Síntese e Escrita) e 8 atividades, em que a atividade 1 referiu-se a estratégia de busca por meio da definição das palavras-chave a serem utilizadas. A atividade 2, realizada em 29 de outubro de 2018, tratou-se da utilização das palavras-chave information professional AND competencies AND innovation, delimitadas no período de 2007 e 2017, buscadas em todo o texto. 
Quanto a atividade 3, o software organizador de bibliografias utilizado foi o EndNote ${ }^{\circledR}$ X7.5 (Bld 9325). Nesta atividade, eliminou-se documentos duplicados, com links e anexos indisponíveis, o que resultou em um total de 887 artigos para análise. Todavia, em decorrência do alto número de documentos para leitura, realizou-se mais uma filtragem com o termo específico information professional nos títulos, palavras-chaves e resumos. Deste modo, chegou-se ao número de 75 artigos.

$\mathrm{Na}$ atividade 4, padronização da seleção dos documentos recuperados, foi realizada a leitura dos títulos, resumos (abstracts) e palavras-chaves de cada documento. Destes, na atividade 5, selecionou-se 73 artigos para serem analisados de acordo com o objetivo estabelecido para este estudo, uma vez que 2 artigos não foram recuperados na integra.

Em relação às atividades 6, 7 e 8 do método SystematicSearchFlow, ou não foram realizadas ou realizou-se parcialmente neste estudo, uma vez que estas fases correspondem a análise, síntese e escrita com a publicação dos resultados do estudo. Isso porque, na atividade 6 são coletados e analisados dados, tais como: artigos e autores mais citados, anos com mais publicações, entre outros. Já nesta investigação esta fase não foi aprofundada, pois se examinou somente os autores com maior número de publicações, o ranking de periódicos científicos, a quantidade de publicações por ano e os artigos mais citados.

\section{Resultados e discussão}

Nesta seção são apresentados os resultados e discussão sobre as competências dos profissionais da informação relacionadas à inovação. Quanto a autoria, houve a repetição de três, as quais estão descritas com seus detalhamentos de quantitativo, instituição e cargo no Quadro 1.

Quadro 1: Autores com maior número de publicações

\begin{tabular}{|l|c|l|}
\hline \multicolumn{1}{|c|}{ Autoria } & Quantidade & \multicolumn{1}{c|}{ Instituição/Cargo } \\
\hline $\begin{array}{l}\text { Giuse, Nunzia } \\
\text { Bettinsoli }\end{array}$ & 2 & $\begin{array}{l}\text { Assistant Vice Chancellor for Knowledge Management; Director, } \\
\text { Eskind Biomedical Library; Professor, Department of Biomedical } \\
\text { Informatics; and Professor, Department of Medicine }\end{array}$ \\
\hline Jain, Priti & 2 & $\begin{array}{l}\text { Department of Library Services, University of Botswana, Gaborone, } \\
\text { Botswana }\end{array}$ \\
\hline $\begin{array}{l}\text { Makori, Elisha } \\
\text { Ondieki }\end{array}$ & 5 & Lecturer, University of Nairobi \\
\hline
\end{tabular}

Fonte: Dados da Pesquisa (2018)

Em relação aos periódicos que aparecem com maior número de artigos publicados, é possível verificar na Figura 1 que a Library Review, a New Library World e a Library Management são os que despontaram com publicações entre cinco e oito. 
Figura 1: Ranking de periódicos científicos

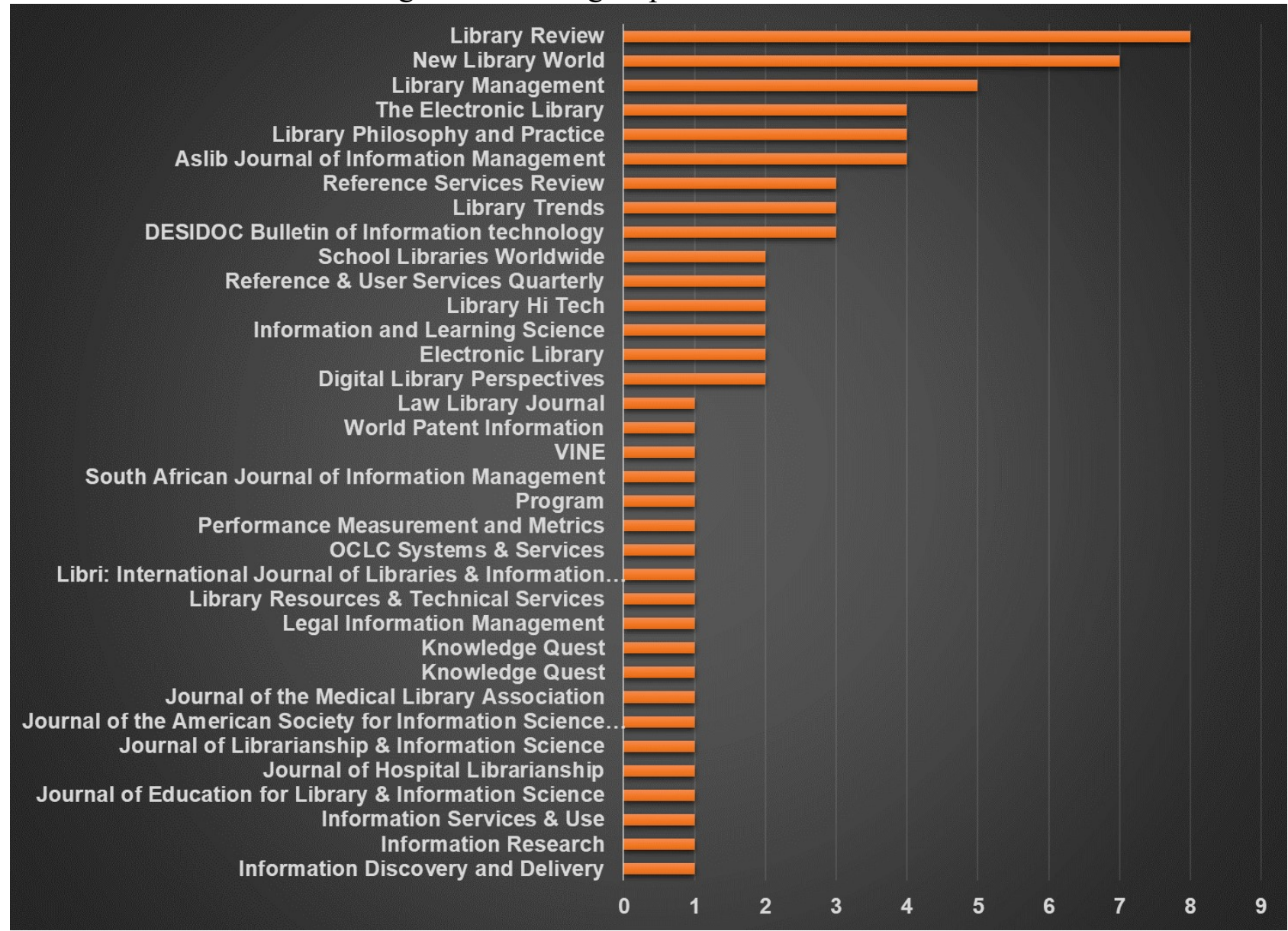

Fonte: Dados da Pesquisa (2018)

Referente ao foco e escopo dos periódicos, estes versam em sua maioria Biblioteconomia, Ciência da Informação e Tecnologia da Informação e Comunicação.

Quanto aos anos das publicações (ver Figura 2), 2017 é o ano com maior número (20) de publicações, seguido dos anos de 2016 (12), 2015 e 2014 (9), e 2012 (1). Ressalta-se a lacuna no ano de 2008, no qual não foram recuperados documentos referentes a relação que se propõe neste estudo. 
Figura 2: Distribuição de artigos por ano de publicação

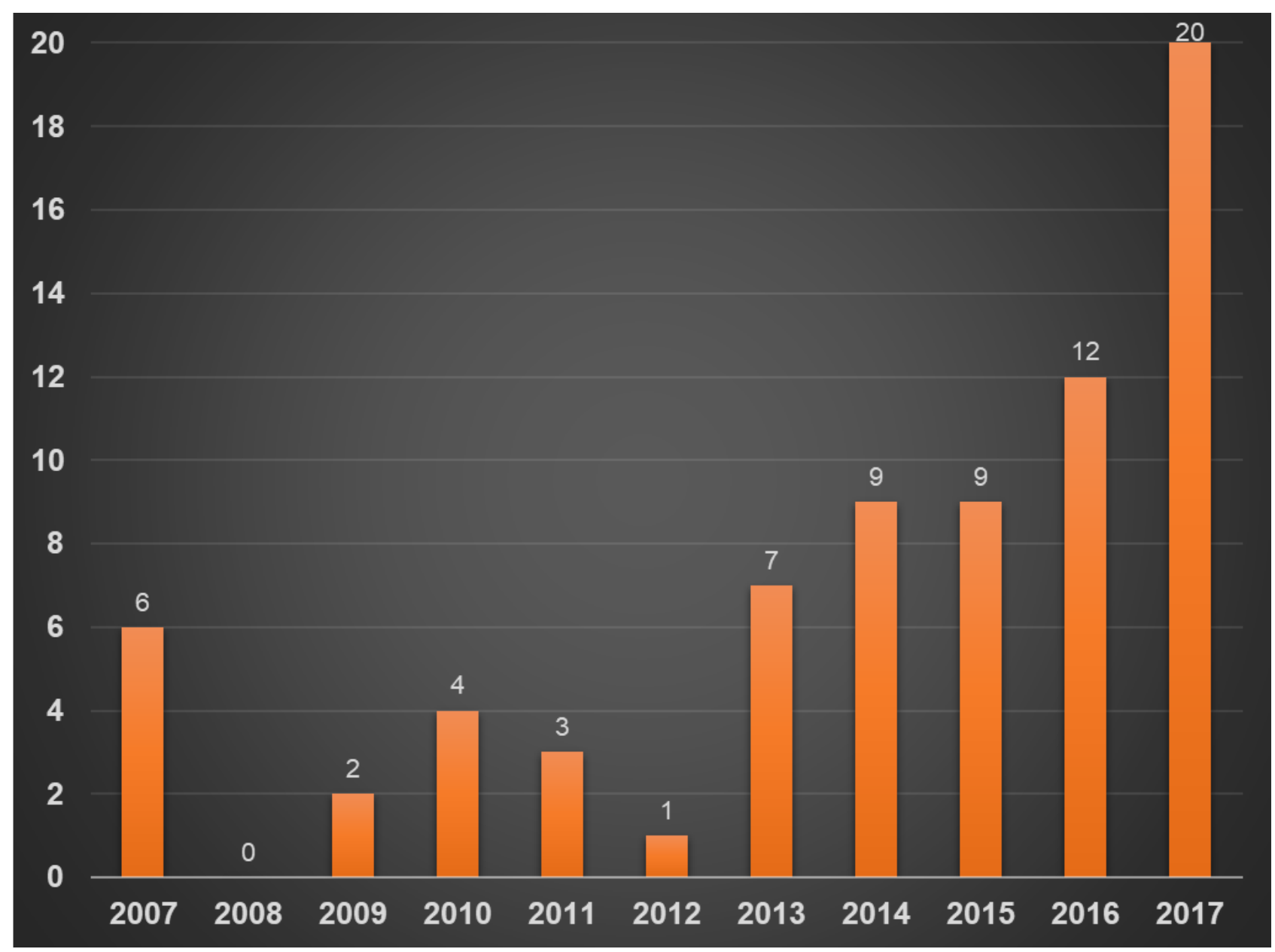

Fonte: Dados da Pesquisa (2018)

Quanto aos artigos com maior número de citações, oito se destacaram por apresentarem entre 49 e 96 citações. Suas temáticas em alguma medida tiveram foco no efeito das inovações tecnológicas nas competências a serem desenvolvidas pelos profissionais da informação, na gestão do conhecimento, na importância do indivíduo e suas necessidades informacionais e, nas oportunidades internacionais e interculturais relacionadas aos currículos de Bibliotecononomia e Ciência da Informação.

Annet Kinengyere (2007) com 96 citações em The effect of information literacy on the utilization of electronic information resources in selected academic and research institutions in Uganda examinou o efeito que o Letramento Informacional (Information Literacy - IL) teve sobre o uso de recursos de informação eletrônica em instituições acadêmicas e de pesquisa em Uganda. Deste modo, focou nas inovações que a Makerere University Library (a maior e mais antiga biblioteca acadêmica de Uganda) empreendeu para garantir que os usuários da biblioteca (a comunidade da Makerere University e outras universidades colaboradoras e instituições de pesquisa em Uganda) fossem treinados sobre como acessar um variedade de recursos de informações disponíveis, avaliar as informações e aplicá-las para atender às suas necessidades. Indicou a IL como uma competência essencial para o século XXI. Abordou sobre as bibliotecas em universidades e instituições de pesquisa em Uganda que ofereciam uma gama de oportunidades para funcionários e estudantes. Todos eles desenvolveram programas para sessões de habilidades informacionais com base em suas necessidades individuais. 
Partridge, Lee e Munro (2010) com 81 citações em Becoming" Librarian 2.0": the skills, knowledge, and attributes required by library and information science professionals in a Web 2.0 world (and beyond) apresentaram uma discussão sobre um projeto de pesquisa que visou preencher a lacuna de como profissionais de biblioteconomia e ciência da informação (LIS) poderiam se tornar "bibliotecários 2.0. Financiado pelo Australian Learning and Teaching Council, o projeto identificou as principais habilidades, conhecimentos e atributos exigidos pelo "bibliotecário 2.0".

Jain (2007) com 72 citações em An empirical study of knowledge management in academic libraries in East and Southern Africa objetivou distinguir gestão da informação (MI) e gestão do conhecimento (GC) e apresentar os resultados da pesquisa em uma pequena escala realizada para investigar práticas de GC em bibliotecas acadêmicas na África Oriental e do Sul. Abordou a questão dos profissionais da biblioteconomia serem resistentes a ir além da caixinha da informação, analisando e interpretando conteúdo. Bem como tratou da importância da identificação de especialização, uma vez que muito desse tipo de conhecimento é tácito e não explícito. Nota-se nesse estudo que a criatividade e inovação foram abordadas como centro da Gestão do Conhecimento.

Kronenfeld, M., et al. (2007) com 60 citações em Review for librarians of evidence-based practice in nursing and the allied health professions in the United States forneceu uma visão geral do estado da prática baseada em evidências (PBE) em enfermagem e profissões de saúde selecionadas e uma sinopse das tendências atuais em incorporar a PBE na educação clínica e na prática nesses campos. Esta visão geral destinou-se a preparar melhor os bibliotecários com uma compreensão geral dos campos e recursos de informação relevantes. Segundo os autores, as habilidades dos bibliotecários em pesquisar, organizar e avaliar informações podem contribuir para promover o desenvolvimento da PBE em uma determinada profissão.

Park, Lu e Marion (2009) com 59 citações em Cataloging professionals in the digital environment: A content analysis of job description avaliaram o estado atual de responsabilidades e conjuntos de habilidades exigidos dos profissionais de catalogação. Identificaram papéis e competências emergentes com foco no ambiente digital e os relacionaram com o conhecimento estabelecido dos padrões e práticas tradicionais de catalogação.

Abdullahi, Kajberg e Virkus (2007) com 58 citações em Internationalization of LIS education in Europe and North America ressaltaram a importância das oportunidades internacionais e interculturais em servir como componentes essenciais na educação e formação de profissionais de bibliotecas e informação. Mais especificamente, forneceram uma visão geral das questões e tendências na internacionalização da educação superior (ES) em geral e ilustraram como o conceito e as abordagens da internacionalização afetaram os ambientes e programas educacionais da biblioteca e ciência da informação (LIS) principalmente na Europa e nos EUA.

Han e Hswe (2010) com 55 citações em The Evolving Role of the Metadata Librarian: Competencies Found in Job Descriptions examinaram as descrições de trabalho de 2000 a 2008 que foram apresentadas em anúncios para bibliotecários de metadados e bibliotecários de catalogação, para determinar onde esses dois papéis convergem e divergem, e o que esses pontos comuns e diferenças transmitem sobre o papel dos bibliotecários de metadados hoje.

Melchionda (2007) com 49 citações em Librarians in the age of the internet: their attitudes and roles apresentou uma revisão das discussões e percepções dos papéis dos bibliotecários na literatura dos últimos dez anos, no contexto do impacto da internet em sua vida profissional. Foi encontrado na revisão da literatura evidências de atitudes contrastantes por parte dos bibliotecários em relação à imposição de tecnologias em rede e digitais no cenário da informação. $\mathrm{Na}$ era da internet, os bibliotecários precisam entrar em acordo com um ambiente de trabalho que é sempre mais desmaterializado e com os novos hábitos de informação de seus usuários. Destacaram a relevância do equilíbrio entre os papéis tradicionais e novos papéis, a fim de deixar sua categoria profissional sobreviver e continuar a ser uma parte crucial e vital na sociedade da informação. 
Ressalta-se que, os periódicos Library Review e New Library destacados entre os com mais publicações também aparecem como as fontes dos artigos mais citados, o que permite inferir que demonstram disseminar o desenvolvimento teórico prático da área.

\section{Considerações finais}

Diante do que foi apresentado nesta pesquisa, verifica-se que os pesquisadores por meio de seus estudos têm realizado esforços para em alguma medida embasar as práticas dos profissionais da informação. Em sua maioria, nos estudos que foram analisados, nota-se a preocupação com a expansão das competências profissionais ante a dinâmica inovativa e tecnológica da sociedade contemporânea.

Em contrapartida, identifica-se também que embora tratem da pertinência dos profissionais serem inovadores não são encontradas ou formuladas teorias a esse respeito. O que será discutido de forma mais detalhadas nos subsequentes artigos a partir desta comunicação.

\section{Agradecimentos}

À Coordenação de Aperfeiçoamento de Pessoal de Nível Superior (CAPES) do Brasil por financiar a bolsa de estudos do doutorado em andamento.

\section{Referências}

Abdullahi, I., Kajberg, L., \& Virkus, S. (2007). Internationalization of LIS education in Europe and North America. New Library World, 108(1/2), 7-24. Recuperado em 29 outubro, 2018, de https://www.emeraldinsight.com/doi/full/10.1108/03074800710722144

Annet Kinengyere, A. (2007). The effect of information literacy on the utilization of electronic information resources in selected academic and research institutions in Uganda. The Electronic Library, 25(3), 328-341. Recuperado em 29 outubro, 2018, de https://www.emeraldinsight.com/doi/full/10.1108/02640470710754832

Ferenhof, H. A., \& Fernandes, R. F. (2016). Desmistificando a revisão de literatura como base para redação científica: método SFF. Revista $A C B$, 21(3), 550-563. Recuperado em 7 janeiro, 2019, de https://revista.acbsc.org.br/racb/article/view/1194/pdf

Han, M. J., \& Hswe, P. (2010). The evolving role of the metadata librarian: competencies found in job descriptions. Library Resources \& Technical Services, 54(3), 129-142. Recuperado em 29 outubro, 2018, de https://go.galegroup.com/ps/i.do? $\mathrm{p}=\mathrm{AONE} \& \mathrm{sw}=\mathrm{w} \& \mathrm{u}=$ googlescholar\&v=2.1\&it=r\&id=GALE\%7C A232175567\&sid= googleScholar\&asid $=$ cbb804b3

Jain, P. (2007). An empirical study of knowledge management in academic libraries in East and Southern Africa. Library review, 56(5), 377-392. Recuperado em 29 outubro, 2018, de https://www.emeraldinsight.com/doi/full/10.1108/00242530710750572

Kronenfeld, M., et al. (2007). Review for librarians of evidence-based practice in nursing and the allied health professions in the United States. Journal of the Medical Library Association:

$J M L A, 95(4), 394$. Recuperado em 29 outubro, 2018, de

https://www.ncbi.nlm.nih.gov/pmc/articles/PMC2000778/ 
Library \& Information Science Abstracts (LISA). Recuperado em 29 outubro, 2018, de https://search.proquest.com/lisa

Library, Information Science and Technology Abstracts (LISTA) Recuperado em 29 outubro, 2018, de https://www.ebsco.com/products/research-databases/library-information-science-andtechnology-abstracts

Melchionda, M. G. (2007). Librarians in the age of the internet: their attitudes and roles: a literature review. New Library World, 108(3-4), 123-140. Recuperado em 29 outubro, 2018, de https://www.ingentaconnect.com/content/mcb/072/2007/00000108/F0020003/art00002

Park, J. R., Lu, C., \& Marion, L. (2009). Cataloging professionals in the digital environment: A content analysis of job descriptions. Journal of the American society for information science and technology, 60(4), 844-857. Recuperado em 29 outubro, 2018, de https://onlinelibrary.wiley.com/doi/abs/10.1002/asi.21007

Partridge, H., Lee, J., \& Munro, C. (2010). Becoming" Librarian 2.0": the skills, knowledge, and attributes required by library and information science professionals in a Web 2.0 world (and beyond). Library Trends, 59(1), 315-335. Recuperado em 29 outubro, 2018, de https://muse.jhu.edu/article/407820/summary

Scopus. Recuperado em 29 outubro, 2018, de https://www.scopus.com/search/form.uri?display=basic

Web of science (WoS). Recuperado em 29 outubro, 2018, de https://webofknowledge.com 